\title{
Pro-inflammatory delipidizing cytokines reduce adiponectin secretion from human adipocytes without affecting adiponectin oligomerization
}

\author{
Peter J Simons, Petra S van den Pangaart ${ }^{1}$, Johannes M F G Aerts ${ }^{2}$ and Louis Boon \\ Department of Cell Biology, Bioceros BV, Yalelaan 46, 3584 CM Utrecht, The Netherlands \\ Departments of ${ }^{1}$ Experimental and Internal Medicine and ${ }^{2}$ Medical Biochemistry, Academic Medical Center, University of Amsterdam, Meibergdreef 15, 1105 \\ AZ Amsterdam, The Netherlands \\ (Requests for offprints should be addressed to P J Simons; Email: p.simons@bioceros.com)
}

\begin{abstract}
Adiponectin and, especially, its oligomeric complex composition have been suggested to be critical in determining insulin sensitivity. Pro-inflammatory cytokines play an important role in the development of insulin resistance in obesity and associated diseases. Therefore, we investigated the effect of long-term exposure of tumour necrosis factor (TNF) $-\alpha$, interleukin (IL)-6, IL-1 $\beta$, and interferon (IFN) $\gamma$ on total insulin-sensitizing adiponectin secretion and adiponectin complex formation from human adipocytes. In parallel, adipocyte delipidation and leptin production levels were monitored. The present study demonstrates that TNF- $\alpha$, IL- $1 \beta$, and IFN- $\gamma$ dose and time dependently suppressed total adiponectin secretion within 7 days $(60,70$, and $35 \%$ reduction respectively). IL-6 was also able to reduce $(50 \%)$ adiponectin production, although only in combination with exogenous soluble IL-6 receptors (sIL-6R). However, the oligomeric distribution (high, middle, and low
\end{abstract}

molecular weight (HMW) complexes) of secreted adiponectin was not altered by any of these cytokines. All studied proinflammatory cytokines resulted in delipidation and reduction of lipid-laden adipocyte numbers. Despite this reduction of lipid-laden adipocytes, TNF- $\alpha$, IL-6/sIL-6R, and IL-1 $\beta$ stimulated leptin release. Our data indicate that (i) long-term pro-inflammatory cytokine exposure downregulates total adiponectin secretion from delipidizing adipocytes and (ii) pro-inflammatory cytokines are not important regulators of adipocyte-derived adiponectin oligomerization. Hence, their individual contribution to low expression of HMWadiponectin found in insulin-resistant conditions seems unlikely. Furthermore, delipidizing adipocytes and preadipocytes are active leptin producers when stimulated by TNF- $\alpha$, IL-6/sIL$6 \mathrm{R}$, and IL-1 $\beta$.

Journal of Endocrinology (2007) 192, 289-299

\section{Introduction}

Adiponectin is a hormone that is produced predominantly by adipocytes (Scherer et al. 1995), and has been shown to have anti-diabetic, anti-atherogenic, and anti-inflammatory properties (Trujillo \& Scherer 2005). In addition, serum levels of adiponectin are reduced in obesity, coronary artery disease, and insulin-resistant and type 2 diabetic patients (Trujillo \& Scherer 2005). Lowered serum adiponectin can also act as a predictor of future development of insulin resistance, type 2 diabetes, and cardiovascular disease (Trujillo \& Scherer 2005).

Adiponectin protein $(30 \mathrm{kDa})$ structurally belongs to the complement C1q-like family, which comprises of a carboxylterminal globular region and an amino-terminal collagenous region (Scherer et al. 1995). Adiponectin in its basic form is a trimer $(90 \mathrm{kDa})$, also called low molecular weight (LMW) adiponectin. Two trimeric subunits associate (via disulphide bonds within the collagen stalk) to hexamers $(180 \mathrm{kDa})$ or middle molecular weight (MMW) adiponectin. These MMW oligomers further assemble into bouquet-like 12-18meric (>360 kDa) high molecular weight (HMW) adiponectin.
Recent studies have suggested that the various oligomeric adiponectin isoforms activate different signaling pathways and exert different functions (Trujillo \& Scherer 2005). Importantly, the ratio of HMW:total adiponectin, but not absolute levels of adiponectin, has been proposed to correlate with insulin sensitivity in mice and humans (Pajvani et al. 2004). Accordingly, a selective reduction in HMW adiponectin was observed in insulin-resistant type 2 diabetic and coronary heart disease patients (Kobayashi et al. 2004, Pajvani et al. 2004). In addition, HMW adiponectin was demonstrated as the most active form in modulating hepatic insulin sensitivity, since only the HMW oligomers decreased glucose levels in adiponectin knockout mice (Pajvani et al. 2004). The vascular protective property of adiponectin has also been reported to be restricted to the HMW component (Kobayashi et al. 2004). In contrast, lipid oxidation and glucose uptake in muscle seem to be attributed to LMW adiponectin or a proteolytic cleavage product thereof, the so-called globular head fragment (Trujillo \& Scherer 2005).

Although the involvement of adiponectin in the development of insulin resistance is now emerging, little is known 
about the factors that regulate the adiponectin oligomeric complex formation in adipocytes. The insulin-sensitizing drug thiazolidinedione enhanced circulating total and HMW adiponectin levels in healthy volunteers and diabetic patients (Pajvani et al. 2004). Furthermore, testosterone selectively suppressed the secretion of total and HMW adiponectin from adipocytes, which explains the sexual dimorphism in serum adiponectin oligomer profiles, i.e. females having higher serum levels of total and HMW adiponectin than males (Xu et al. 2005). Since multiple pro-inflammatory cytokines, produced by either adipocytes or stromavascular cells and macrophages within adipose tissue, contribute to the chronic low-grade inflammatory state found in obesity and related diseases (Lyon et al. 2003, Schmidt \& Duncan 2003), we investigated the possible role of long-term exposure of tumour necrosis factor (TNF)- $\alpha$, interleukin (IL)-6, IL-1 $\beta$, and interferon (IFN) $-\gamma$ on the secretion of total adiponectin and its oligomers from human in vitro differentiated adipocytes.

\section{Materials and Methods}

\section{Subjects}

Subcutaneous white adipose tissue samples were obtained from healthy 29- to 52-year-old women (43 \pm 9 years, mean \pm s.D.; $n=5$ ) undergoing elective liposuction surgery in thighs/hips. The body mass index (BMI) range of the donors was between 19 and $36 \mathrm{~kg} / \mathrm{m}^{2}\left(28 \pm 7 \mathrm{~kg} / \mathrm{m}^{2}\right.$, mean \pm s.D.; two lean, one overweight, and two obese donors). All women were otherwise healthy and free of metabolic (e.g. lipid storage disorder or diabetes) or immune diseases. Informed consent was obtained from each participating donor by Zen-Bio, Inc., Research Triangle Park, NC, USA (see Cell culture).

\section{Cell culture}

Frozen purified subcutaneous white adipocyte tissue-derived preadipocytes (see Subjects) were purchased from Zen-Bio. These preadipocyte preparations (passage 2) were devoid of endothelial cells and macrophages as exemplified by negative staining for vascular endothelial growth factor receptors and macrophage markers (CD14 and CD68) respectively. Generation of mature lipid-storing adipocytes from these preadipocytes was performed as described previously (Simons et al. 2005). Briefly, preadipocytes were thawed and suspended in Dulbecco's modified Eagle's medium (DMEM)/Ham's F12 medium (17.5 mM D-glucose) supplemented with $10 \%(\mathrm{v} / \mathrm{v})$ fetal calf serum (FCS), $15 \mathrm{mM}$ HEPES and antibiotics (preadipocyte medium from Zen-Bio), and subsequently seeded in 96-well flat-bottomed culture plates (CostarCorning, Schiphol-Rijk, The Netherlands). After reaching $100 \%$ confluence, preadipocytes were put on differentiation medium (Zen-Bio) containing DMEM/Ham's F12 supplemented with $3 \%(\mathrm{v} / \mathrm{v})$ FCS, $15 \mathrm{mM}$ HEPES, $1 \mu \mathrm{M}$ dexamethasone, $100 \mathrm{nM}$ human insulin, $250 \mu \mathrm{M}$ isobutyl methylxathine (IBMX), $10 \mu \mathrm{M}$ proprietary peroxisome proliferator-activated receptor- $\gamma(\operatorname{PPAR}-\gamma)$ agonist (an indomethacin-derived compound), $33 \mu \mathrm{M}$ D-biotin, $17 \mu \mathrm{M}$ potassium pantothenate, and antibiotics to initiate adipocyte differentiation. After a 3-day initiation period, differentiation medium was replaced by adipocyte medium (Zen-Bio) containing the same substances as described for differentiation medium, but devoid of IBMX and PPAR- $\gamma$ agonist. Every 2-3 days, cells were fed with fresh adipocyte medium. After 14 days, preadipocytes were fully differentiated into adipocytes (40-80\% of surface area was covered with adipocytes, see Fig. 2). These adipocytes were gently washed five times with adipocyte medium, and subsequently replenished (referred to as day 0 ) with adipocyte medium with or without recombinant IFN- $\gamma$ (U-Cy-Tech, Utrecht, The Netherlands), IL-1 $\beta$ (PeproTech Ltd, London, UK), IL-6 (PeproTech), or TNF- $\alpha$ (PeproTech) at various concentrations (as indicated in the Results section and figures). In some experiments, IL-6 $(10 \mathrm{ng} / \mathrm{ml})$ was also studied in the presence of $100 \mathrm{ng} / \mathrm{ml}$ soluble recombinant human IL-6 receptors (sIL-6R; RDI Research Diagnostics, Inc., Concord, MA, USA). On days 3 and 5 , the adipocyte cultures were fed with fresh adipocyte medium supplemented with corresponding cytokines as described previously. Harvested supernatants (on days 3, 5, and 7) were stored at $-20^{\circ} \mathrm{C}$ for total adiponectin and its complexes, and leptin analysis (see below). Fully differentiated adipocytes were cultured for a total period of 7 days in the presence of cytokines, and each condition was performed in triplicate. On day 7, two independent observers (P V D P and $\mathrm{P}$ J S) counted the numbers of adipocytes (characterized by round/oval cells completely filled with lipid droplets) in each well, and these are expressed as adipocyte numbers $/ \mathrm{cm}^{2}$.

\section{Quantification of lipid accumulation by Oil Red O staining}

The Oil Red O staining method was performed as described previously (Ramirez-Zacarias et al. 1992) with slight modifications. On day 7, cells were rinsed with PBS (BioWhittaker-Cambrex, Verviers, Belgium), fixed with $3 \cdot 7 \%(\mathrm{v} / \mathrm{v})$ formaldehyde (Sigma Europe) in PBS for $1 \mathrm{~h}$, and stained with filtrated $0 \cdot 6 \%(\mathrm{w} / \mathrm{v})$ Oil Red O dye (Sigma) in $60 \%(\mathrm{v} / \mathrm{v})$ isopropanol (ICN, Zoetermeer, The Netherlands) for $1.5 \mathrm{~h}(40 \mu \mathrm{l} /$ well $)$. Hereafter, wells were extensively washed with water, and excess of water was evaporated at $60{ }^{\circ} \mathrm{C}$ for $1 \mathrm{~h}$. Oil Red $\mathrm{O}$ dye in differentiated adipocytes was extracted by adding 100\% isopropanol $(100 \mu \mathrm{l} /$ well $)$ for $10 \mathrm{~min}$, and the optical density of this solution was measured at a wavelength of $490 \mathrm{~nm}$ (and referenced at $655 \mathrm{~nm}$ ).

\section{Adiponectin and leptin ELISA measurements}

Total adiponectin and leptin levels in culture supernatants were determined by using specific ELISAs (from R\&D Systems, Inc., Minneapolis, MN, USA, and from Biosource 
International, Inc., Camarillo, CA, USA respectively) according to the manufacturer's protocols.

\section{Separation of adiponectin complexes}

Culture supernatants were diluted in 'adipocyte medium' (see Cell culture) resulting in equal absolute amounts of total adiponectin protein (as determined by ELISA) in order to determine possible effects of cytokines on the relative adiponectin isoform distribution within each donor. These diluted supernatants were incubated in a non-reducing NuPAGE SDS sample buffer (Invitrogen) at room temperature (RT) or at $95^{\circ} \mathrm{C}$ (to establish specificity of adiponectin bands, see Results) for 10 min as described previously (Waki et al. 2003), and subsequently, electrophoretically separated by a NuPAGE 4-12\% gradient Bis-Tris SDS/PAGE gel (Invitrogen) using NuPAGE morpholinepropanesulfonic acid running buffer (Invitrogen). Molecular weight standards (BlueRanger from Pierce, Rockford, IL, USA) were run in parallel. After transfer of proteins onto nitrocellulose and a $5 \%(\mathrm{w} / \mathrm{v})$ BSA blocking step, the adiponectin complexes were detected using biotinylated goat anti-human adiponectin polyclonal antibodies (R\&D Systems; $0 \cdot 2 \mu \mathrm{g} / \mathrm{ml} \mathrm{IgG} \mathrm{in} \mathrm{PBS/5 \%} \mathrm{BSA/0.05 \%} \mathrm{(v/v)}$ Tween 20 , overnight at $4{ }^{\circ} \mathrm{C}$ ), followed by a streptavidin polyhorseradish peroxidase step (Sanquin, Amsterdam, The Netherlands; $10 \mathrm{ng} / \mathrm{ml}$ streptavidin in PBS/5\% BSA $/ 0 \cdot 05 \%$ Tween 20, $1 \mathrm{~h}$ at RT). Immunoblots were exposed to $\mathrm{X}$-ray film using SuperSignal West Pico chemiluminescent substrate (sensitivity in the low picogram region) according to the manufacturer's instructions (Pierce Biotechnology).

\section{Cell proliferation assay}

The proliferative rate of cells, which were cultured under various conditions (see above, Cell culture), was established using a bromodeoxyuridine (BrdU) incorporation ELISA (Roche Molecular Biochemicals). Briefly, on 'day 6' after initiating cytokine treatments, $10 \mu \mathrm{M}$ BrdU (Roche) was added to the cells. After an overnight labeling period, cells were fixed and DNA denatured in FixDenat solution (Roche). Subsequently, BrdU incorporation was determined by adding anti-BrdU antibodies conjugated to peroxidase (Roche). Immune complexes were detected by a subsequent substrate/chemiluminescence reaction $\left(\mathrm{H}_{2} \mathrm{O}_{2}\right.$, luminal, and 4-iodophenol; Roche) in a luminometer (Perkin \& Elmer, Boston, MA, USA).

\section{Statistical analysis}

The numbers of independent experiments are given in the figure legends. Data are expressed as means \pm s.D. Because of inter-individual variation of adiponectin production found in adipocyte cultures from studied donors $(n=5)$, adiponectin data are presented as percentages of adiponectin production with cytokines when compared with corresponding control adipocyte cultures without cytokines (set at 100\%) for each time point (Figs 3 and 4). Using GraphPad Instat software, the ANOVA was conducted to check whether differences among experimental groups were significant (adiponectin results were logarithmically transformed to obtain Gaussian distribution). The TukeyKramer multiple comparison post test was conducted to identify differences between two experimental treatments. In both the cases, $P$ values $<0 \cdot 05$ were considered statistically significant.

\section{Results}

Effect of pro-inflammatory cytokines on lipid-containing adipocyte numbers and lipid storage

All investigated pro-inflammatory cytokines (at $10 \mathrm{ng} / \mathrm{ml}$ ), with the exception of IL-6, resulted in decreased lipid-laden
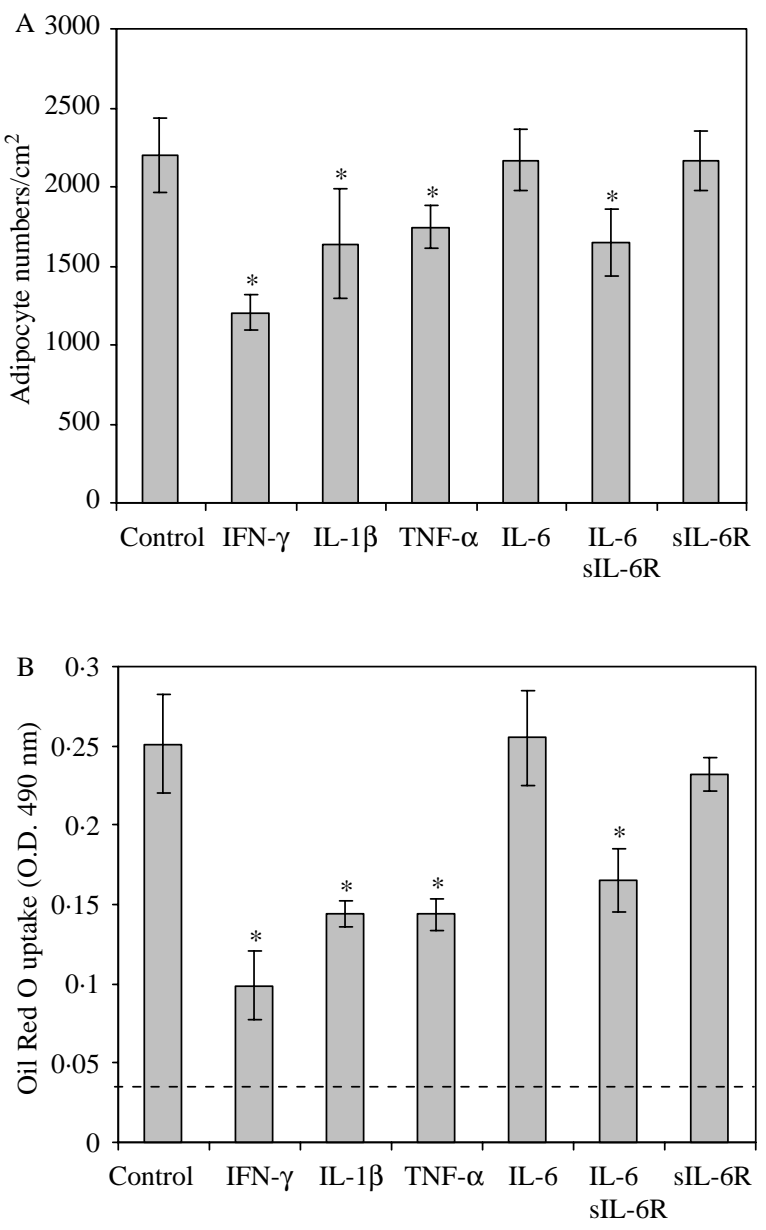

Figure 1 Effect of pro-inflammatory cytokines (at $10 \mathrm{ng} / \mathrm{ml}$; TNF- $\alpha$ $(n=5), \mathrm{IL}-1 \beta(n=5), \mathrm{IFN}-\gamma(n=3), \mathrm{IL}-6(n=4), \mathrm{IL}-6 / \mathrm{sIL}-6 \mathrm{R}(n=3))$ and sIL-6R (at $100 \mathrm{ng} / \mathrm{ml} ; n=3$ ) on adipocyte numbers (A) and lipid accumulation (B) on day 7 . Dashed line represents background Oil Red O staining in cultures containing confluent preadipocytes only. Values are means \pm s.D. for three to five independent subjects. Each condition was tested in triplicate. Asterisk $(*)$ means significantly different when compared with control adipocytes without addition of cytokines, $P<0 \cdot 05$ (ANOVA and Tukey-Kramer's test). 
adipocyte numbers and triglyceride contents after an exposure time of 7 days (Fig. 1A and B respectively). However, the effect of these cytokines was relatively modest on adipocyte number reduction (ranging from 20 to $45 \%$ reduction in comparison with non-treated adipocytes), while delipidation was much more pronounced (ranging from 45 to $70 \%$ reduction). Interestingly, IFN $-\gamma$ was the most potent factor to induce delipidation, and resulted in fibroblast-like cells containing relatively large lipid droplets (when compared with control adipocytes, which were typically round to oval cells containing multiple lipid droplets; Fig. 2). TNF- $\alpha$ and
IL-1 $\beta$ treatment both showed elongated adipocytes (Fig. 2). In addition, preadipocyte cell densities seemed to be enhanced after TNF- $\alpha$ and IL-1 $\beta$ exposure (see Effect of pro-inflammatory cytokines on preadipocyte proliferation). Noteworthy, these TNF- $\alpha-$, IL-1 $\beta-$, and IFN- $\gamma$-mediated effects were dose-dependent, since 1 and $100 \mathrm{ng} / \mathrm{ml}$ gave similar results as found with $10 \mathrm{ng} / \mathrm{ml}$, but gradually disappeared after exposure to lower cytokine concentrations (i.e. $0 \cdot 1,0 \cdot 01$, and $0 \cdot 001 \mathrm{ng} / \mathrm{ml}$; data not shown). Endogenous TNF- $\alpha$, IL- $1 \beta$, and IFN- $\gamma$ concentrations were always below $0.01 \mathrm{ng} / \mathrm{ml}$ in control adipocyte cultures.
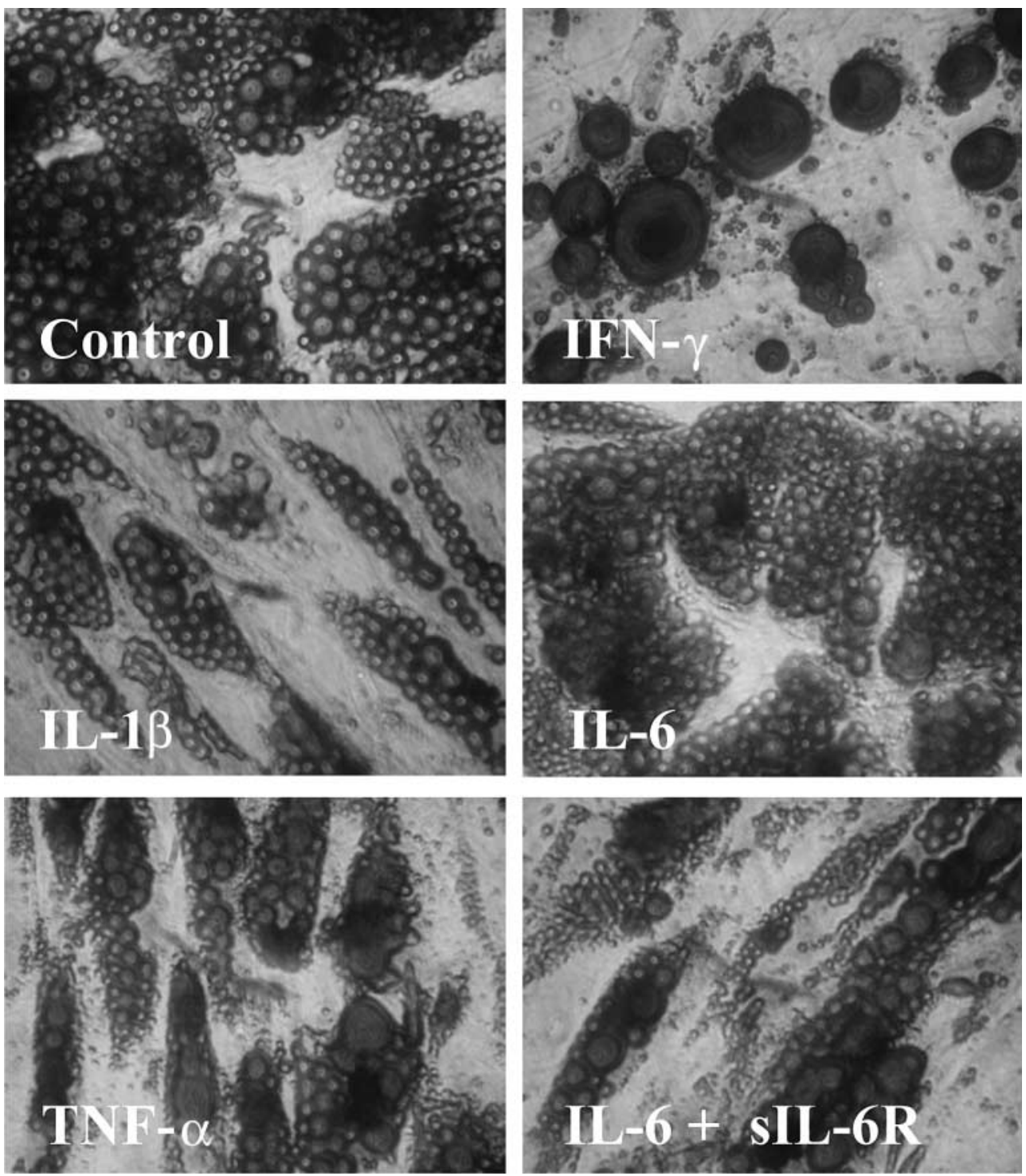

Figure 2 Effect of pro-inflammatory cytokines $(10 \mathrm{ng} / \mathrm{ml})$ on adipocyte morphology and lipid (Oil Red $\mathrm{O} /$ hematoxylin staining) accumulation on day 7 . Note the elongated cell morphology after TNF- $\alpha(n=5)$, IL-1 $\beta(n=5)$ and IL-6/sIL-6R $(n=3)$ treatment, and the large lipid droplets after IFN- $\gamma(n=3)$ treatment in comparison with round/oval control adipocytes without addition of cytokines or with IL-6 $(n=4)$. Results are representative for three to five independent subjects. Each condition was tested in triplicate. Magnification, $\times 400$. 
As stated previously, IL-6 demonstrated no effect on adipocyte delipidation. However, when adipocytes were treated with IL-6 (10 ng/ml) plus sIL-6R (100 ng/ml), adipocyte numbers and lipid contents decreased (Fig. 1) in a similar manner as observed with TNF- $\alpha$ and IL-1 $\beta$. Furthermore, adipocyte morphology after IL-6/sIL-6R treatment also resembled that of TNF- $\alpha$ - and of IL-1 $\beta$-treated adipocytes (i.e. elongated adipocytes and higher preadipocyte numbers; see Fig. 2). sIL-6R alone did not result in adipocyte delipidation, although low endogenous IL-6 levels (range: $\sim 0 \cdot 025-0 \cdot 1 \mathrm{ng} / \mathrm{ml}$ ) were detected in control adipocytes.

Pro-inflammatory cytokines dose and time dependently suppress total adiponectin secretion

Differentiated control adipocytes gradually increased their total adiponectin secretion levels during a culture period of 7 days (Fig. 3A). We observed a considerable inter-donor variation of adiponectin production (five donors were used in our study, and a range of approximately $10-400 \mathrm{ng} / \mathrm{ml}$ adiponectin on day 7 was found). This inter-donor variation was not related to age or BMI, or to adipocyte numbers or lipid accumulation.

TNF- $\alpha$, IL- $1 \beta$, and IFN- $\gamma$ dose and time dependently inhibited total adiponectin production levels (Fig. 3). TNF- $\alpha$ and IL-1 $\beta$ already showed a significant inhibition of $\sim 30-35 \%$ within 3 -days of culture (TNF- $\alpha$ at $10 \mathrm{ng} / \mathrm{ml}$, and IL-1 $\beta$ at $0 \cdot 1$ and $10 \mathrm{ng} / \mathrm{ml}$ ). After 7 days of exposure to TNF- $\alpha$ and IL-1 $\beta$, these inhibitory levels increased to $\sim 50-70 \%$. Rather surprisingly, IFN- $\gamma$ (at $10 \mathrm{ng} / \mathrm{ml}$ ) resulted in maximum adiponectin suppression of $\sim 35 \%$ after 7 days, despite its above-described superior effect on delipidation. Moreover, 1 and $100 \mathrm{ng} / \mathrm{ml}$ TNF- $\alpha$, IL- $1 \beta$, or IFN- $\gamma$ demonstrated a similar decrement of adiponectin secretion when compared with corresponding cytokines at $10 \mathrm{ng} / \mathrm{ml}$; lower concentrations $(0 \cdot 1,0 \cdot 01$, and $0 \cdot 001 \mathrm{ng} / \mathrm{ml})$ were less effective or ineffective (Fig. 3; data not shown).
A

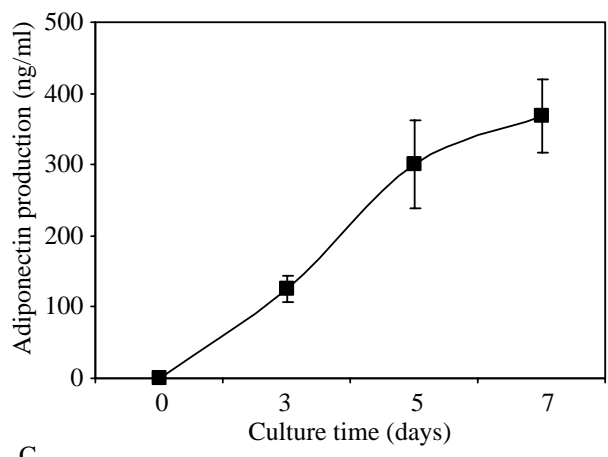

$\mathrm{C}$

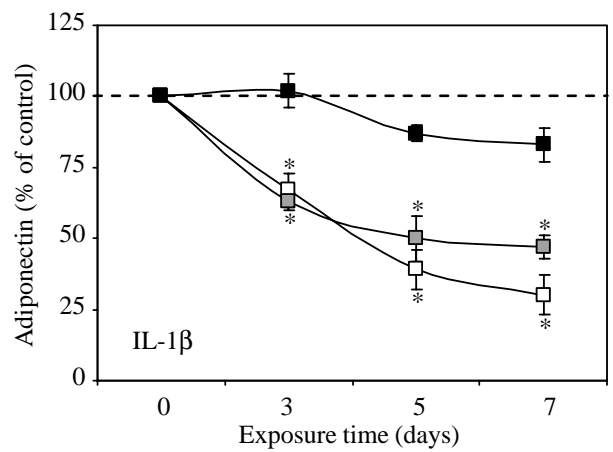

B

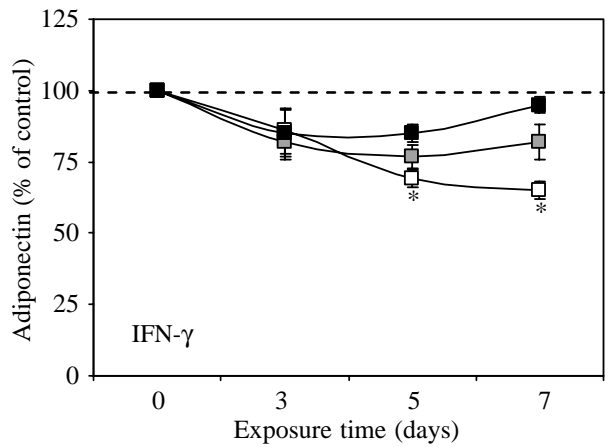

$\mathrm{D}$

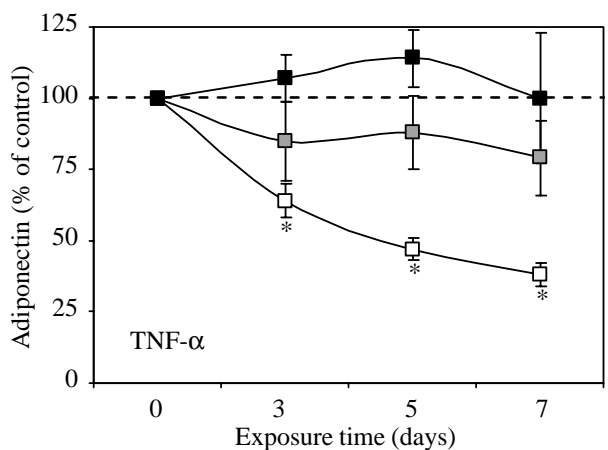

Figure 3 Dose- and time-dependent inhibitory effects of pro-inflammatory cytokines on total adiponectin secretion from adipocytes. (A) The kinetics of adiponectin production by control adipocytes from one representative subject is shown (of in total five independent subjects studied). (B-D) Because of inter-donor variation, adiponectin data are presented as percentages of adiponectin production with cytokines when compared with corresponding control adipocyte cultures without cytokines (set at $100 \%$ at each time point) in each separate experiment (ranges of adiponectin levels in control adipocyte cultures from five subjects were: $2 \cdot 9-126 \cdot 0 \mathrm{ng} / \mathrm{ml}$ at day $3 ; 4 \cdot 7-300 \cdot 0 \mathrm{ng} / \mathrm{ml}$ at day $5 ; 6 \cdot 0-424 \cdot 0 \mathrm{ng} / \mathrm{ml}$ at day 7$)$. Adiponectin release from adipocytes was inhibited after IFN- $\gamma(\mathrm{B}), \mathrm{IL}-1 \beta(\mathrm{C})$, and TNF- $\alpha(\mathrm{D})$ exposure. Cytokine added at: white boxes, $10 \mathrm{ng} / \mathrm{ml}(n=5-6)$; grey boxes, $0 \cdot 1 \mathrm{ng} / \mathrm{ml}(n=3)$; black boxes, $0 \cdot 001 \mathrm{ng} / \mathrm{ml}(n=2)$. Values are means \pm s.D. for two to five independent subjects (B-D). Each condition was tested in triplicate. Asterisk $\left({ }^{*}\right)$ means significantly different when compared with control adipocytes without addition of cytokines on the same day, $P<0 \cdot 05$ (ANOVA and Tukey-Kramer's test). 
IL-6 (0.001-100 ng/ml) could not influence adiponectin release from adipocytes (at $10 \mathrm{ng} / \mathrm{ml}$, see Fig. 4A; at all other concentrations; data not shown), however, the combination of IL-6 $(10 \mathrm{ng} / \mathrm{ml})$ and sIL-6R $(100 \mathrm{ng} / \mathrm{ml})$ resulted in an adiponectin suppression of $\sim 40-50 \%$ on days 5 and 7 (see Fig. 4A). When sIL-6R was added to adipocytes, $\sim 20 \%$ $(P>0 \cdot 05)$ inhibition of adiponection secretion was shown on day 7 (Fig. 4A), which probably reflected the effect of low endogenous IL-6 production in our adipocyte culture system (and also indicated dose-dependency of the IL-6/sIL-6R effect).

Altogether, delipidizing cytokines - TNF- $\alpha$, IL-6/sIL-6R, IL-1 $\beta$, and IFN- $\gamma$ - clearly suppressed the release of total adiponectin from adipocytes after long-term exposure, i.e. longer than 3 days.

Pro-inflammatory cytokines do not influence adiponectin oligomeric complex release

In order to determine a possible effect of pro-inflammatory delipidizing cytokines on adiponectin oligomerization, we used
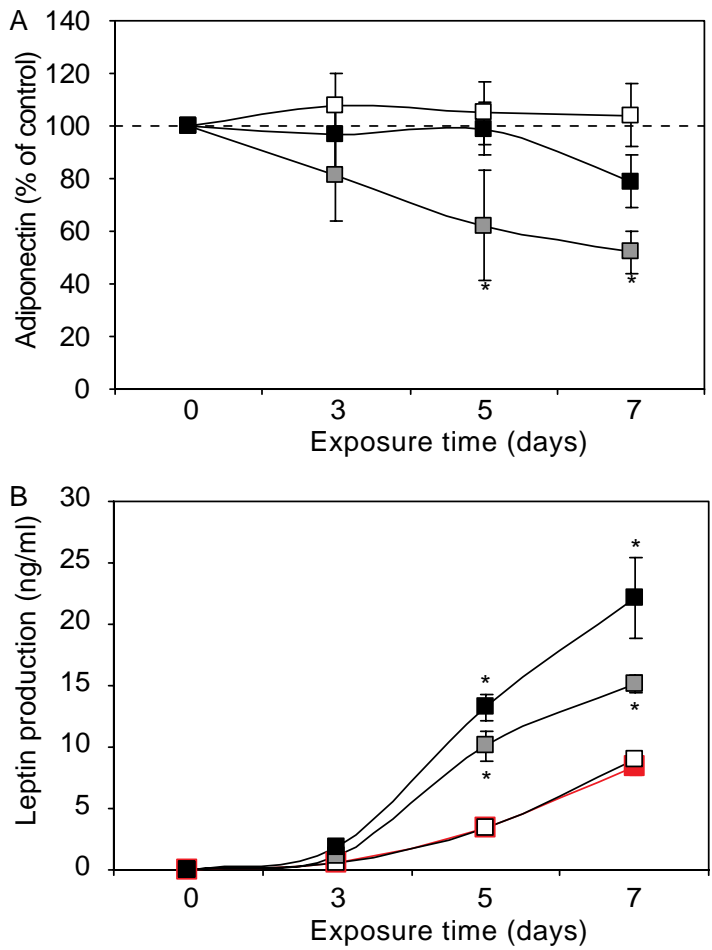

Figure 4 Effects of IL-6 $(10 \mathrm{ng} / \mathrm{ml})$ and slL-6R $(100 \mathrm{ng} / \mathrm{ml})$ on adiponectin (A) and on leptin (B) production from (pre)adipocytes. Red boxes, control adipocytes $(n=4)$; white boxes, IL-6 $(n=4)$; grey boxes, IL-6 plus sIL-6R $(n=3)$; black boxes, sIL-6R $(n=3)$. Adiponectin data are presented as percentages of adiponectin production with IL-6 and/or sIL-6R when compared with corresponding control adipocyte cultures without IL-6 and/or sIL-6R, see Fig. 3 for explanation. Values are means \pm s.D. for three to four independent subjects. Each condition was tested in triplicate. Asterisk $\left.{ }^{*}\right)$ means significantly different when compared with control adipocyte cultures without addition of IL-6 and/or sIL-6R on the same day, $P<0 \cdot 05$ (ANOVA and Tukey-Kramer's test). the method of Waki et al. (2003). As shown in Fig. 5A, multiple adiponectin isoforms were secreted by control adipocytes on day 5, which comprised dimeric, trimeric, tetrameric, pentameric, hexameric, and HMW adiponectin oligomeric complexes. Furthermore, trimeric LMW adiponectin was the most abundantly released isoform. The aforementioned adiponectin oligomer profile was found using in vitro differentiated adipocytes from all five tested donors (data not shown). Analysis of adiponectin containing supernatants collected on days 3 and 7 gave identical outcomes $(n=3$; data not shown), when compared with day 5.

Although investigated pro-inflammatory cytokines inhibited total adiponectin levels from adipocytes, TNF- $\alpha$, IL$1 \beta$, and IFN- $\gamma$ (all at $10 \mathrm{ng} / \mathrm{ml}$ ) were not able to change the relative secretory adiponectin oligomer composition (Fig. 5A). This ineffectiveness on adiponectin oligomerization was donor-independent (data not shown). Moreover, adiponectin profiles after cytokine exposure were found to be identical when days 3, 5, and 7 supernatants were compared (data not shown). The specificity of adiponectin signals was also investigated: denaturated samples (see Materials and Methods section) resulted in two typical adiponectin bands (Waki et al. 2003, Richards et al. 2006b), one migrated to $\sim 60 \mathrm{kDa}$ and the other to $\sim 30 \mathrm{kDa}$, which represented dimeric and monomeric adiponectin respectively (Fig. 5B).

Treatment of differentiated adipocytes with IL-6 $(10 \mathrm{ng} / \mathrm{ml}$; Fig. 5A), sIL-6R (100 ng/ml), or a combination of both factors (data not shown) did not show any effect on the adiponectin oligomer distribution (irrespective of tested time point or donor).

\section{Effect of pro-inflammatory cytokines on leptin secretion}

Since we have previously reported a leptin-enhancing effect of TNF- $\alpha$ and IL-1 $\beta$ on preadipocytes (Simons et al. 2005), the influence of various pro-inflammatory cytokines on leptin production was also monitored in adipocyte cultures. Our adipocyte cultures consisted of a mixture of preadipocytes and lipid-loaded adipocytes (typically $40-80 \%$ of surface area was covered with adipocytes on day 0 ). As expected, increasing levels of leptin were found in control adipocyte cultures during a 7 -day culture period (Fig. 6). TNF- $\alpha$ and IL-1 $\beta$, however, dose and time dependently enhanced leptin secretion $(\sim 200$ and $\sim 400 \%$ increment on day 7 respectively; Fig. $6 \mathrm{~B}$ and $\mathrm{C}$ ) by a mixed population of delipidizing adipocytes and preadipocytes (see above, Effect of proinflammatory cytokines on lipid-containing adipocyte numbers and lipid storage). IFN- $\gamma$ suppressed leptin production from these cultures $(\sim 60 \%$ inhibition; Fig. 6A). IL-6 alone showed no effect on leptin release, but IL-6/sIL-6R resulted in an upregulation of leptin secretion (Fig. 4B). sIL-6R also increased leptin levels (Fig. 4B), again, exemplifying the activity of endogenously synthesized IL-6. 


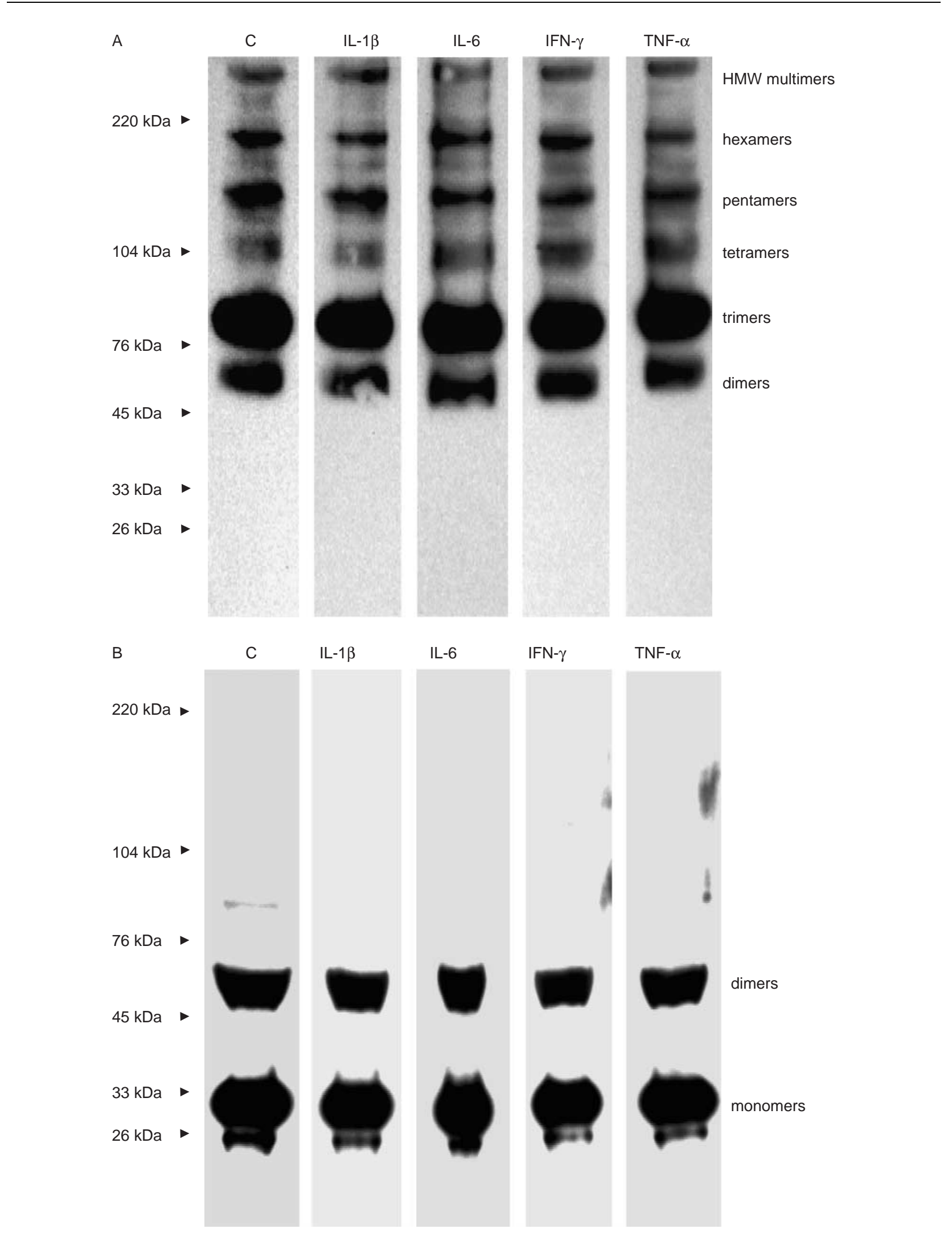


Effect of pro-inflammatory cytokines on preadipocyte proliferation

TNF- $\alpha$, IL- $1 \beta$, and IFN- $\gamma$ induced low proliferative responses (IL-1 $\beta$ and TNF- $\alpha>$ IFN- $\gamma$; all at $10 \mathrm{ng} / \mathrm{ml}$ ) from preadipocytes, and, most probably, from fully delipidized adipocytes (see Fig. 7). These results are in agreement with our previous work (Simons et al. 2005), which showed that confluent, growtharrested preadipocytes re-entered the S-phase of the cell cycle during pro-inflammatory cytokine treatment. Further, IL-6 (at $10 \mathrm{ng} / \mathrm{ml}$; see Fig. 7) and sIL-6R (at $100 \mathrm{ng} / \mathrm{ml}$ ) demonstrated no mitogenic effect, whereas the combination of IL- 6 and sIL$6 \mathrm{R}$ resulted in a BrdU labeling comparable with IL-1 $\beta$ and TNF- $\alpha$ exposure (data not shown).

\section{Discussion}

Obesity is defined as an excess of adipose tissue, and is a strong risk factor for development of insulin resistance, hypertension, type 2 diabetes, and cardiovascular disease (Lyon et al. 2003, Schmidt \& Duncan 2003). Obese animals and individuals typically express increased levels of pro-inflammatory TNF- $\alpha$, IL-6, and leptin, and, in contrast, decreased levels of anti-inflammatory insulin-sensitizing adiponectin. Additionally, intra-adipose macrophage infiltration has been described to be elevated in obesity (Weisberg et al. 2003). Obesity is therefore considered as a chronic and low-grade inflammatory condition that leads to insulin resistance, and subsequently to the above-mentioned diseases. Diet-induced weight loss in obese individuals results in lowering of macrophage infiltration (Bruun et al. 2006) and of TNF- $\alpha$, IL-6, and leptin levels (Lyon et al. 2003), whereas total and HMW adiponectin levels increase (Kobayashi et al. 2004, Bobbert et al. 2005). Particularly, low HMW adiponectin oligomer expression is suggested to play an important role in the development of insulin resistance (see Introduction).

While many studies have investigated factors that influence total adiponectin secretion levels, e.g. thiazolidinedione (Pajvani et al. 2004), testosterone (Xu et al. 2005), dexamethasone (Degawa-Yamauchi et al. 2005), TNF- $\alpha$ (Kappes \& Loffler 2000, Bruun et al. 2003, Degawa-Yamauchi et al. 2005, Wang et al. 2005), and IL-6 (Bruun et al. 2003), only a few studies have addressed their effect on secretory adiponectin complexes (Pajvani et al. 2004, Xu et al. 2005, Bodles et al. 2006). This prompted us to examine the effect of pro-inflammatory cytokines, including those that are mplicated in the chronic inflammatory obese status, on total adiponectin production and its oligomeric complexes from human adipocytes. Although plasma levels of pro-inflammatory cytokines are generally very low (below $0 \cdot 1-0 \cdot 01 \mathrm{ng} / \mathrm{ml}$; Hotamisligil et al. 1995, Sopasakis et al. 2004), local intra-adipose tissue TNF- $\alpha$ and IL-6 concentrations are known to be potentially high $(>1 \mathrm{ng} / \mathrm{ml}$; Hotamisligil et al. 1995, Sopasakis et al. 2004), hence, conceivably reaching concentrations used in our study. We clearly showed that long-term TNF- $\alpha$, IL-6/sIL-6R, IL-1 $\beta$, and IFN $-\gamma$ treatment gradually suppressed total adiponectin release from human adipocytes. The strong TNF- $\alpha$-induced adiponectin inhibition found by us seems to be in contrast to what has been reported by others (Degawa-Yamauchi et al. 2005, Wang et al. 2005). However, in these studies, human in vitro differentiated or ex vivo isolated adipocytes were treated with TNF- $\alpha$ for only short periods of time (i.e. 24-48 h), which resulted in minimal $(\sim 7 \%)$ to no reduction of adiponectin secretion. In contrast, adipocyte-derived adiponectin mRNA levels were found to be suppressed within 24-48 h after TNF- $\alpha$ exposure in two independent studies (Bruun et al. 2003, Wang et al. 2005), and Kappes et al. demonstrated strong inhibition $(\sim 50 \%)$ of adiponectin protein release from TNF- $\alpha$-treated human adipocytes after $48 \mathrm{~h}$. These discrepancies may be the result of different culture techniques. Nonetheless, our data favor the idea that long-term TNF- $\alpha$ exposure downregulates total adiponectin secretion by human adipocytes. In addition, IL-6 alone was unable to influence adiponectin synthesis, and the presence of sIL-6R was obligatory in order to demonstrate IL-6 bioactivity in human adipocytes (see also below, leptin secretion and adipocyte delipidation). This observation is in agreement with another study (Bruun et al. 2003) using human adipocytes: adiponectin gene expression (adiponectin protein secretion was, however, not investigated) was reduced by the IL-6/sIL-6R combination within $48 \mathrm{~h}$, whereas IL-6 was ineffective.

To evaluate whether pro-inflammatory cytokine-induced decrement of total adiponectin is associated with a change of adiponectin oligomeric profiles, we studied adiponectin oligomers in supernatants from our adipocyte cultures. Control adipocytes produced multiple adiponectin isoforms, which were detected using the electrophoresis/blotting technique initially described by Waki et al. (2003). Apart from the commonly detected trimeric, hexameric, and HMW adiponectin (Scherer et al. 1995, Tsao et al. 2002, Waki et al. 2003, Xu et al. 2005, Richards et al. 2006a,b), we also observed dimers, tetramers, and pentamers. This adiponectin profile was found in all studied donors. After denaturation, only monomeric and dimeric adiponectin isoforms were observed, a phenomenon that evidenced adiponectin specificity of all

Figure 5 Effect of pro-inflammatory cytokines $(10 \mathrm{ng} / \mathrm{ml})$ on the relative adiponectin oligomer distribution secreted from adipocytes on day 5. Equal absolute amounts of total adiponectin protein were loaded onto a $4-12 \%$ gradient Bis-Tris SDS/PAGE gel and electrophoretically separated, and then immunoblotted using polyclonal antibodies against human adiponectin, see Materials and Methods. (A) Under nonheating and non-reducing conditions, dimeric, trimeric, tetrameric, pentameric, hexameric, and $\mathrm{HMW}$ multimeric adiponectin isoforms were detected in supernatants from control adipocytes. IL-1 $\beta$, IL- 6 , IFN- $\gamma$, and TNF- $\alpha$ did not affect the relative distribution of adiponectin oligomers. (B) Heating of these supernatants resulted in adiponectin monomers and dimers. A representative experiment from one subject is shown (of in total five independent subjects studied). C, control. 

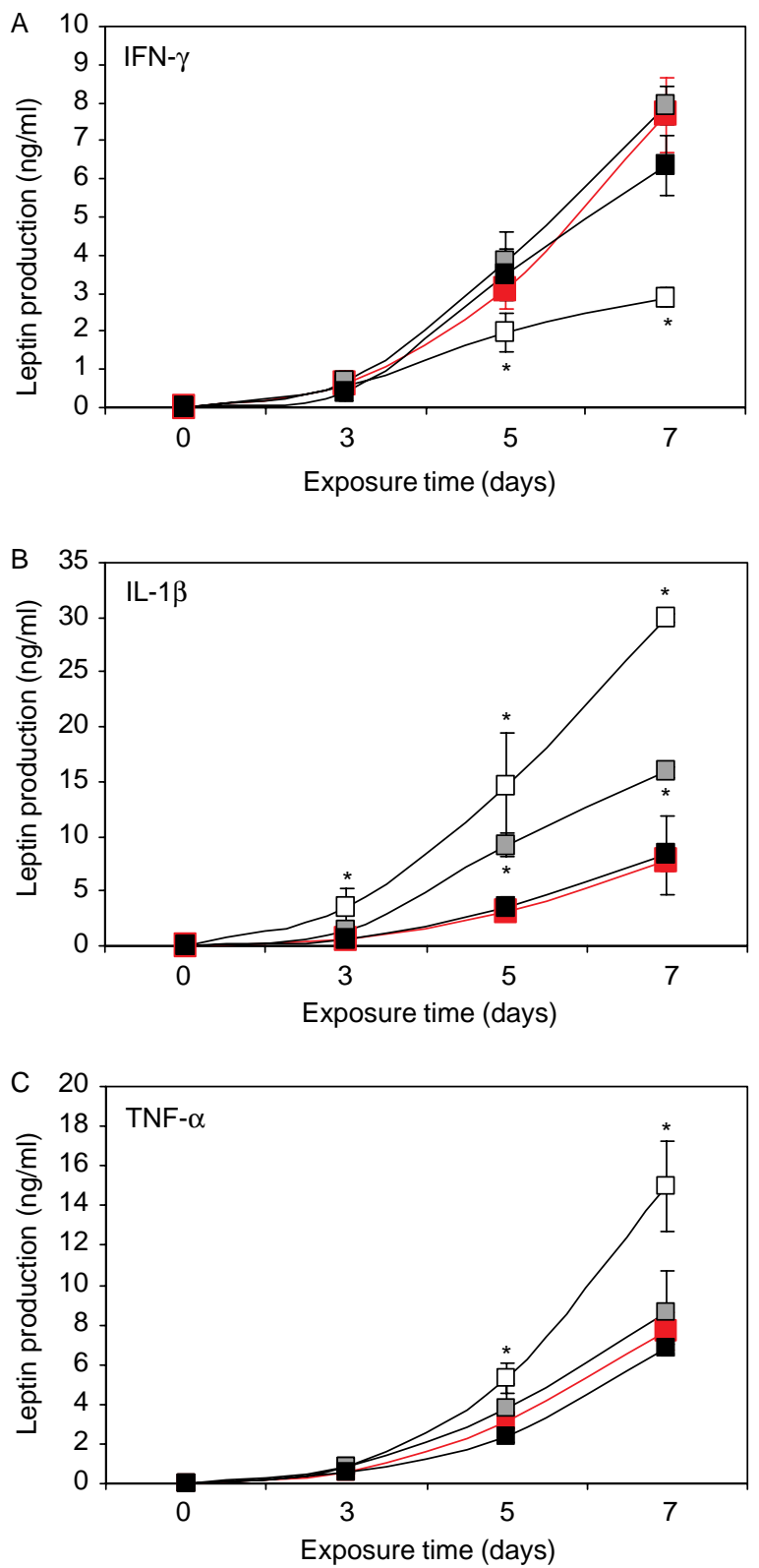

Figure 6 Dose- and time-dependent effects of pro-inflammatory cytokines on leptin secretion from (pre)adipocytes. IFN- $\gamma$ suppressed (A), and both IL-1 $\beta$ (B) and TNF- $\alpha$ (C) stimulated leptin production from preadipocytes and delipidizing adipocytes. Cytokine added at: white boxes, $10 \mathrm{ng} / \mathrm{ml}(n=5)$; grey boxes, $0 \cdot 1 \mathrm{ng} / \mathrm{ml}(n=3)$; black boxes, $0 \cdot 001 \mathrm{ng} / \mathrm{ml}(n=2)$. Values are means \pm s.D. for two to five independent subjects. Each condition was tested in triplicate. Asterisk $\left(^{*}\right)$ means significantly different when compared with control cell cultures without addition of cytokines on the same day (red boxes), $P<0 \cdot 05$ (ANOVA and Tukey-Kramer's test). bands in our blotting experiments (Waki et al. 2003, Richards et al. 2006b). Apparently, the usage of our polyclonal antiadiponectin antibody in combination with a highly sensitive chemiluminescent substrate Super Signal facilitated recognition of dimeric, tetrameric, and pentameric adiponectin isoforms. Interestingly, $\left[{ }^{35} \mathrm{~S}\right]$ methionine/cysteine-labeled 3T3-L1 adipocytes also revealed secretory dimeric, tetrameric, and pentameric adiponectin after immunoprecipitation (Scherer et al. 1995). In accordance with the previous studies (Tsao et al. 2002, Richards et al. 2006a,b), trimeric adiponectin oligomers were the most predominant adiponectin isoforms that were secreted from in vitro differentiated adipocytes. Regardless of the adiponectin distribution found in our study, long-term exposure to TNF- $\alpha$, IL-6/sIL-6R, IL-1 $\beta$, and IFN $-\gamma$ could not change adiponectin oligomer composition from human adipocytes.

We confirmed previous in vitro studies on TNF- $\alpha$-induced adipocyte delipidation (reviewed in Sethi \& Hotamisligil 1999). Additionally, we demonstrated similar effects after IL-6/sIL-6R, IL- $1 \beta$, and IFN- $\gamma$ exposure. The latter factor, however, resulted in a dissimilar adipocyte morphology than former factors, which indicates differential mechanisms of action. Although not the scope of our study, future specific analysis on adipocyte insulin signaling and on adipogenic and apoptotic markers could be helpful to determine whether these cytokines exhibit merely anti-insulin activity (i.e. induction of lipolysis/suppression of lipogenesis in fully differentiated adipocytes) or induce a disputed process called dedifferentiation (i.e. the capacity of differentiated lipid-laden adipocytes to revert to undifferentiated lipid-free fibroblastlike preadipocytes; Fernyhough et al. 2006), or apoptosis. Nevertheless, all these pro-inflammatory cytokines stimulated immature preadipocyte proliferation. Moreover, high leptin

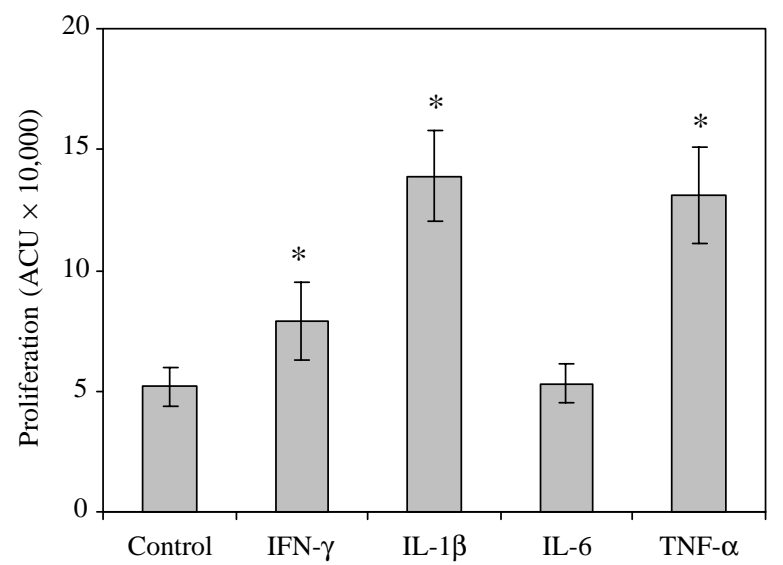

Figure 7 Effect of pro-inflammatory cytokines $(10 \mathrm{ng} / \mathrm{ml})$ on the proliferative capacity of preadipocytes and delipidized adipocytes on day 7. Proliferation was measured by using BrdU labeling, and is expressed as arbitrary chemiluminescent units (ACU). Values are means \pm s.D. for three independent subjects. Each condition was tested in triplicate. Asterisk $\left(^{*}\right)$ means significantly different when compared with control cell cultures without addition of cytokines, $P<0.05$ (ANOVA and Tukey-Kramer's test). 
levels were detected in our adipocyte cultures (containing a mixture of preadipocytes and delipidizing adipocytes) treated with TNF- $\alpha$, IL-6/sIL-6R, or IL-1 $\beta$, which is in agreement with our recent finding that slowly proliferating undifferentiated preadipocytes are potent leptin producers under these culture conditions (Simons et al. 2005). Most likely, delipidizing adipocytes found in our study also contributed to these high leptin levels. Interestingly, genes that are associated with lipid metabolism were suppressed in adipose tissue obtained from various obese and type 2 diabetes animals (Nadler et al. 2000), while the expression of cell cycle-related genes were found to be raised in obese animals and humans (Nadler et al. 2000, Lee et al. 2005). Further, Cancello et al. (2005) described leptin mRNA expression in dedifferentiated fibroblast-like adipocytes, and concluded that leptin gene expression was independent of cellular lipid content.

In conclusion, we have demonstrated that long-term exposure to TNF- $\alpha$, IL-6/sIL-6R, IL- $1 \beta$, and IFN- $\gamma$ suppresses total adiponectin secretion from delipidizing adipocytes, without affecting the relative distribution of secreted adiponectin isoforms. Simultaneously, preadipocytes slowly expanded and leptin production increased under these circumstances. Based on our findings, we speculate that autocrine/paracrine pro-inflammatory cytokines, in particular obesity-related TNF- $\alpha$ and IL-6, may be directly responsible for low total adipocyte-derived adiponectin plasma levels; however, aberrant adiponectin oligomer profiles in insulin-resistant individuals are probably formed after adiponectin release from adipocytes. This concept contrasts the mechanism of action ascribed to thiazolidinedione and testosterone, which both have direct effects on adiponectin oligomer shifts in adipocytes within $24 \mathrm{~h}$ (Pajvani et al. 2004, Xu et al. 2005). Intriguingly, acute concanavalin A-induced TNF- $\alpha$ stimulation $(<24 \mathrm{~h})$ resulted in a reduction of total circulating adiponectin levels in mice, while adiponectin oligomer profiles remained unaltered (Morris et al. 2006). Evidently, identification of the exact mechanism(s) that controls extracellular bioavailability and clearance of adiponectin complexes by pro-inflammatory cytokines will provide better understanding of the development and treatment of obesity-related insulin resistance.

\section{Acknowledgements}

The authors declare that there is no conflict of interest that would prejudice the impartiality of the scientific work.

\section{References}

Bobbert T, Rochlitz H, Wegewitz U, Akpulat S, Mai K, Weickert MO, Mohlig M, Pfeiffer AF \& Spranger J 2005 Changes of adiponectin oligomer composition by moderate weight reduction. Diabetes 54 2712-2719.
Bodles A, Banga A, Rasouli N, Ono F, Kern PA \& Owens RJ 2006 Pioglitazone increases secretion of high molecular weight adiponectin from adipocytes. American Journal of Physiology. Endocrinology and Metabolism 291 1100-1105.

Bruun JM, Lihn AS, Verdich C, Pedersen SB, Toubro S, Astrup A \& Richelsen B 2003 Regulation of adiponectin by adipose tissue-derived cytokines: in vivo and in vitro investigations in humans. American Journal of Physiology. Endocrinology and Metabolism 285 E527-E533.

Bruun JM, Helge JW, Richelsen B \& Stallknecht B 2006 Diet and exercise reduce low-grade inflammation and macrophage infiltration in adipose tissue but not in skeletal muscle in severely obese subjects. American Journal of Physiology. Endocrinology and Metabolism 290 E961-E967.

Cancello R, Pietri-Rouxel F \& Clement K 2005 Spontaneous lipid accumulation in primary cultures of dedifferentiated human adipocytes. Adipocytes 1 73-78.

Degawa-Yamauchi M, Moss KA, Bovenkerk JE, Shankar SS, Morrison CL, Lelliott CJ, Vidal-Puig A, Jones R \& Considine RV 2005 Regulation of adiponectin expression in human adipocytes: effects of adiposity, glucocorticoids, and tumor necrosis factor alpha. Obesity Research 13 662-669.

Fernyhough ME, Vierck JL \& Dodson MV 2006 Assessing a non-traditional view of adipogenesis: adipocyte dedifferentiation - mountains or molehills? Cells, Tissues, Organs 182 226-228.

Hotamisligil GS, Arner P, Caro JF, Atkinson RL \& Spiegelman BM 1995 Increased adipose tissue expression of tumor necrosis factor-alpha in human obesity and insulin resistance. Journal of Clinical Investigation 95 2409-2415.

Kappes A \& Loffler G 2000 Influences of ionomycin, dibutyryl-cycloAMP and tumour necrosis factor-alpha on intracellular amount and secretion of apM1 in differentiating primary human preadipocytes. Hormone and Metabolic Research 32 548-554.

Kobayashi H, Ouchi N, Kihara S, Walsh K, Kumada M, Abe Y, Funahashi T \& Matsuzawa Y 2004 Selective suppression of endothelial cell apoptosis by the high molecular weight form of adiponectin. Circulation Research 94 e27-e31.

Lee YH, Nair S, Rousseau E, Allison DB, Page GP, Tataranni PA, Bogardus C \& Permana PA 2005 Microarray profiling of isolated abdominal subcutaneous adipocytes from obese vs non-obese Pima Indians: increased expression of inflammation-related genes. Diabetologia 48 1776-1783.

Lyon CJ, Law RE \& Hsueh WA 2003 Minireview: adiposity, inflammation, and atherogenesis. Endocrinology 144 2195-2200.

Morris AM, Sennello JA, Fayad RA, Eckel RH, Dinarello CA \& Fantuzzi G 2006 T cell-mediated hepatic inflammation modulates adiponectin levels in mice: role of tumor necrosis factor alpha. Metabolism 55 555-559.

Nadler ST, Stoehr JP, Schueler KL, Tanimoto G, Yandell BS \& Attie AD 2000 The expression of adipogenic genes is decreased in obesity and diabetes mellitus. PNAS 97 11371-11376.

Pajvani UB, Hawkins M, Combs TP, Rajala MW, Doebber T, Berger JP, Wagner JA, Wu M, Knopps A, Xiang AH et al. 2004 Complex distribution, not absolute amount of adiponectin, correlates with thiazolidinedionemediated improvement in insulin sensitivity. Journal of Biological Chemistry 279 12152-12162.

Ramirez-Zacarias JL, Castro-Munozledo F \& Kuri-Harcuch W 1992 Quantitation of adipose conversion and triglycerides by staining intracytoplasmic lipids with Oil red O. Histochemistry 97 493-497.

Richards AA, Hickman IJ, Wang AY, Jones AL, Newell F, Mowry BJ, Whitehead JP, Prins JB \& Macdonald GA 2006a Olanzapine treatment is associated with reduced high molecular weight adiponectin in serum: a potential mechanism for olanzapine-induced insulin resistance in patients with schizophrenia. Journal of Clinical Psychopharmacology 26 232-237.

Richards AA, Stephens T, Charlton HK, Jones A, Macdonald GA, Prins JB \& Whitehead JP 2006b Adiponectin multimerisation is dependent on conserved lysines in the collagenous domain: evidence for regulation of multimerisation by alterations in post-translational modifications. Molecular Endocrinology 20 1673-1687.

Scherer PE, Williams S, Fogliano M, Baldini G \& Lodish HF 1995 A novel serum protein similar to $\mathrm{C} 1 \mathrm{q}$, produced exclusively in adipocytes. Journal of Biological Chemistry 270 26746-26749.

Schmidt MI \& Duncan BB 2003 Diabesity: an inflammatory metabolic condition. Clinical Chemistry and Laboratory Medicine 41 1120-1130. 
Sethi JK \& Hotamisligil GS 1999 The role of TNF alpha in adipocyte metabolism. Seminars in Cell and Developmental Biology 10 19-29.

Simons PJ, van den Pangaart PS, van Roomen CP, Aerts JM \& Boon L 2005 Cytokine-mediated modulation of leptin and adiponectin secretion during in vitro adipogenesis: evidence that tumor necrosis factor-alpha- and interleukin-1beta-treated human preadipocytes are potent leptin producers. Cytokine 32 94-103.

Sopasakis VR, Sandqvist M, Gustafson B, Hammarstedt A, Schmelz M, Yang X, Jansson PA \& Smith U 2004 High local concentrations and effects on differentiation implicate interleukin-6 as a paracrine regulator. Obesity Research 12 454-460.

Trujillo ME \& Scherer PE 2005 Adiponectin - journey from an adipocyte secretory protein to biomarker of the metabolic syndrome. Journal of Internal Medicine 257 167-175.

Tsao TS, Murrey HE, Hug C, Lee DH \& Lodish HF 2002 Oligomerization state-dependent activation of NF-kappa B signaling pathway by adipocyte complement-related protein of $30 \mathrm{kDa}$ (Acrp30). Journal of Biological Chemistry 277 29359-29362.

Waki H, Yamauchi T, Kamon J, Ito Y, Uchida S, Kita S, Hara K, Hada Y, Vasseur F, Froguel P et al. 2003 Impaired multimerization of human adiponectin mutants associated with diabetes. Molecular structure and multimer formation of adiponectin. Journal of Biological Chemistry $\mathbf{2 7 8}$ 40352-40363.

Wang B, Jenkins JR \& Trayhurn P 2005 Expression and secretion of inflammation-related adipokines by human adipocytes differentiated in culture: integrated response to TNF-alpha. American Journal of Physiology. Endocrinology and Metabolism 288 E731-E740.

Weisberg SP, McCann D, Desai M, Rosenbaum M, Leibel RL \& Ferrante AW Jr 2003 Obesity is associated with macrophage accumulation in adipose tissue. Journal of Clinical Investigation 112 1796-1808.

Xu A, Chan KW, Hoo RL, Wang Y, Tan KC, Zhang J, Chen B, Lam MC, Tse C, Cooper GJ et al. 2005 Testosterone selectively reduces the high molecular weight form of adiponectin by inhibiting its secretion from adipocytes. Journal of Biological Chemistry 280 18073-18080.

Received in final form 7 November 2006

Accepted 21 November 2006 Made available online as an Accepted Preprint 21 November 2006 